\title{
Modelo de simulación del crecimiento de callo óseo en consolidación de fracturas
}

\author{
José Manuel Naveiro Gómez ${ }^{1}$, Jorge Rosell Pradas ${ }^{1}$ \\ ${ }^{1}$ Grupo Biomateriales \\ Instituto de Investigación en Ingeniería de Aragón (I3A) \\ Universidad de Zaragoza, Mariano Esquillor s/n, 50018, Zaragoza, Spain. \\ Tel. +34-976762707, e-mail: jmnaveiro@unizar.es
}

\section{Resumen}

Desarrollo de un modelo de simulación de crecimiento del callo óseo en la consolidación de fracturas, con aplicación a las fracturas femorales tratadas mediante clavos intramedulares. En la bibliografía se puede encontrar que es un problema cada vez más presente [1] y que los enfoques son orientados a remodelación [2].

\section{Material y Métodos}

El trabajo se basa en el análisis de la difusión de diferentes magnitudes (en concreto las MSCs y los condrocitos) que al alcanzar un valor umbral, desencadenan el crecimiento del callo óseo. El algoritmo determina si se produce crecimiento o no en función de tres magnitudes: los umbrales de las dos concentraciones y el volumen que debe crecer según el valor de la velocidad de crecimiento.

Aplicando las ecuaciones de difusión a un modelo con múltiples parámetros, se controla la velocidad de crecimiento. En concreto aplicando la ley de Fick:

$$
\frac{d c}{d t}=\nabla(D \nabla c)
$$

En la figura 1 se ve una clasificación de las partes del modelo y la nomenclatura empleada. El modelo se compone de clavo intramedular, frontera superior, frontera inferior y gap. En los elementos que conforman las fronteras, las caras que no se encuentran conectadas a otros elementos se consideran caras libres.

Las condiciones de contorno son:

$$
\partial \Omega=\Gamma_{1} \cup \Gamma_{2}\left\{\begin{array}{ll}
c(\vec{r}, t)=0 & \Gamma_{1} \text { (condiciones esenciales) } \\
D \nabla c \vec{n}=\mathcal{D} & \Gamma_{2} \text { (condiciones naturales) }
\end{array}\right\}
$$

estando aplicada la primera condición en todo el contorno salvo en la fuente de concentración que se encuentra en la zona más alejada de las fronteras y que correspondería a las condiciones naturales.

Desarrollando la formulación débil del problema y discretizando en intervalos temporales se obtiene la siguiente expresión del problema:

$$
\left(\frac{1}{\Delta t} M+N\right) \bar{a}_{k+1}=\bar{f}+\frac{1}{\Delta t} M \bar{a}_{k}
$$

siendo a el valor nodal de las concentraciones. La velocidad se controla mediante la función:

$$
\varphi(\xi, \eta)=\frac{4 A}{\left[1+e^{-a\left(\xi-\xi_{0}\right)}\right]\left[1+e^{-b\left(\eta-\eta_{0}\right)}\right]}+B
$$

donde $\xi$ y $\xi_{0}$ son la concentración de células mesenquimales y la concentración umbral, $\eta$ y $\eta_{0}$ son la concentración de condrocitos y la concentración umbral de condrocitos. Los valores a y b son reguladores de la velocidad que dependen de las moléculas TNF- $\alpha$, BMP o TGF- $\beta 1$ y que son multiplicadores de la velocidad. Por su parte, A y B son constantes que se ajustan para normalizar la función.

El algoritmo de crecimiento, programado con Fortran 77 [3], permite la creación de malla. Para ello sigue dos reglas: sólo pueden crecer caras libres y no es posible crecer donde había previamente elementos. Con estas dos premisas y tomando la dirección normal a las caras, el callo puede crecer hasta cerrar el gap de la fractura.

Finalmente, para el flujo de cálculos y la regulación del proceso iterativo para obtener el volumen en un intervalo de tiempo, se empleó Python [4] como lenguaje de control.

\section{Resultados}

Los resultados del algoritmo de crecimiento se pueden ver en la figura 2. La figura muestra una fractura transversa con clavo intramedular y como

Revista "Jornada de Jóvenes Investigadores del I3A", vol. 8 (Actas de la IX Jornada de Jóvenes Investigadores del I3A -11 de diciembre de 2020). ISSN 2341-4790. 
crece el callo a lo largo del tiempo el tiempo. Las concentraciones van avanzando hasta el foco de fractura donde, cumpliendo con los umbrales y la velocidad límite, el algoritmo permite crecer a la malla hasta cerrar los dos frentes. La figura 3 muestra la evolución del callo óseo en 3 dimensiones y se puede comparar la situación final con la imagen radiológica de la fractura real (figura 4)

\section{Conclusiones}

Se ha propuesto un algoritmo de crecimiento de callo que permita hacer una evaluación predictiva más acorde a la evidencia clínica, haciendo crecer el callo en función de las magnitudes biológicas involucradas en el problema. El enfoque y los algoritmos desarrollados suponen un avance significativo a lo existente en la bibliografía, ya que por una parte incluyen magnitudes biológicas involucradas en el problema, y por otrageneran la nueva malla de manera automática en las zonas de crecimiento, sin ninguna restricción previa.

\section{FIGURAS / LEYENDAS}

EJE FEMORAL

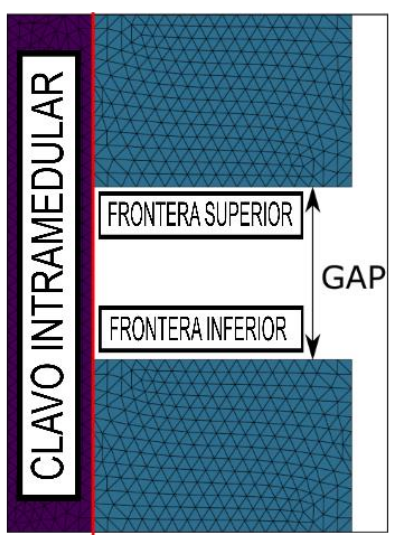

CARAS LIBRES

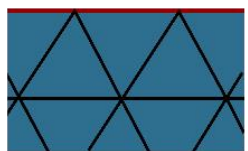

Figura 1. Conceptos de la fractura

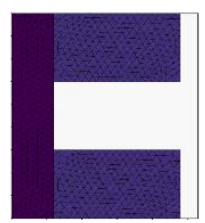

INSTANTE INICIAL

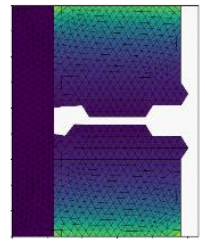

DÍA 18

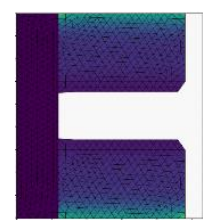

DIA 2

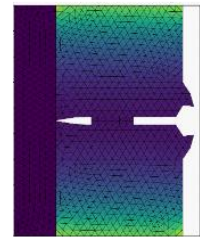

DÍA 28

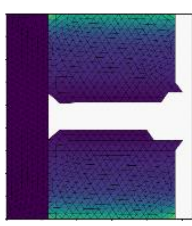

DÍA 6

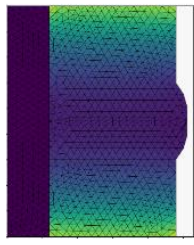

DÍA 35
Figura 2. Cierre de fractura

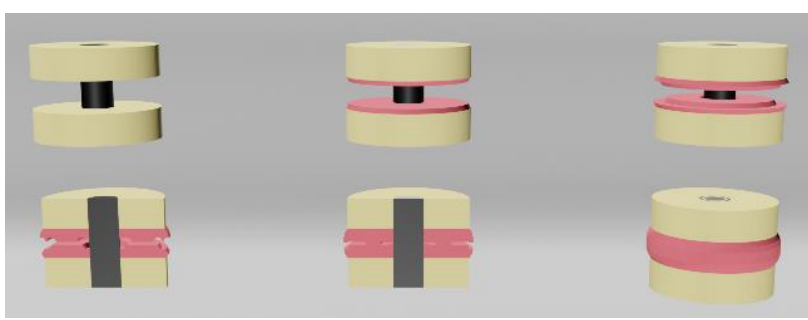

Figura 3. Vista 3D de los cierres de fractura

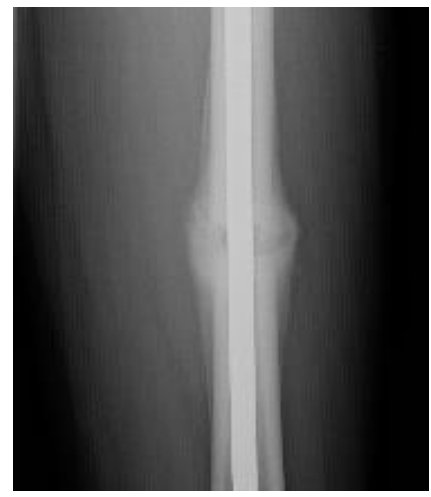

Figura 4. Radiografía de callo de fractura

\section{REFERENCIAS}

[1]. Roberts SE, Goldacre MJ. Time trends and demography of mortality after fractured neck of femur in an English population, 1968-98: database study. BMJ.2003Oct4;327(7418):771-5.doi: 10.1136/bmj. 327.7418.771.PMID:14525871; PMCID: PMC214070.GOLDBERG, K. and KEHOE, B. Cloud robotics and automation: A survey of related work. Berkeley: EECS Department, University of California Berkeley, 2013. Technical Report.

[2]. Wang M, Yang N, Wang X. A review of computational models of bone fracture healing. Med Biol Eng Comput. 2017 Nov;55(11):1895-1914. doi: 10.1007/s11517-017-1701-3. Epub 2017 Aug 8. PMID: 28785849.

[3]. gfortran-the GNU Fortran compiler, part of GCC, version 8 . available at https://gcc.gnu.org/wiki/GFortranBinaries

[4]. Python Software Foundation. Python Language Reference, version 3.8. Available at http://www.python.org 